\title{
Automatic segmentation of the lumen in magnetic resonance images of the carotid artery
}

\author{
Danilo Samuel Jodas, Aledir Silveira Pereira and João Manuel R. S. Tavares
}

\begin{abstract}
The segmentation of the lumen and vessel wall in Magnetic Resonance (MR) images of carotid arteries represents a crucial step towards the evaluation of cerebrovascular diseases. However, the automatic segmentation of the lumen is still difficult due to the usual low quality of the images and the presence of elements that might affect the accuracy of the results. In this article, we describe a fully automatic method to identify the location of the lumen in MR images of the carotid artery. A circularity index is used to assess the roundness of the regions identified by the K-means algorithm in order to obtain the one with the maximum value, which represents the potential lumen region. The boundary of the identified lumen region is then refined by an active contour algorithm. The proposed method achieved a maximum Dice coefficient of $0.91 \pm 0.04$ and $0.74 \pm 0.16$ in 181 postcontrast 3DT1-weighted and 181 proton density-weighted MR images, respectively. Therefore, the method seems to be promising for identifying the correct location of the lumen in MR images.
\end{abstract}

Danilo Samuel Jodas (Corresponding author)

CAPES Foundation - Ministry of Education of Brazil, Brasilia - DF, 70040-020, Brazil

Instituto de Ciência e Inovação em Engenharia Mecânica e Engenharia Industrial, Faculdade de Engenharia, Universidade do Porto, Rua Dr. Roberto Frias, s/n, 4200-465, Porto, Portugal, e-mail: danilojodas@gmail.com

Aledir Silveira Pereira

Universidade Estadual Paulista "Júlio de Mesquita Filho“, Rua Cristóvão Colombo, 2265, 15054 000, S. J. do Rio Preto, Brazil, e-mail: aledir@ sjrp.unesp.br

João Manuel R. S. Tavares

Instituto de Ciência e Inovação em Engenharia Mecânica e Engenharia Industrial, Departamento de Engenharia Mecânica, Faculdade de Engenharia, Universidade do Porto, Rua Dr. Roberto Frias, s/n, 4200-465, Porto, Portugal, e-mail: tavares@fe.up.pt 


\section{Introduction}

The identification of structures in medical images represents an important step towards the assessment of pathologies related to several diseases. In particular, the segmentation of the arterial system in images acquired from the well-known imaging modalities allows the detection and evaluation of cardiovascular diseases $[1,2]$. The most common cardiovascular disease is the atherosclerosis, which is still the most dangerous disease that affects the majority of people in the world. The accumulation of fatty material and cholesterol in the walls of the arterial system is the primary condition to the appearance of atherosclerosis, which reduces the normal blood flow through the artery and, consequently, leads to events such as heart attacks and strokes $[3,4,5]$.

The advent of novel imaging-based systems contributed to increase the accuracy in detecting and evaluating the pathologies associated to cardiovascular diseases. Ultrasound, computed tomography angiography (CTA) and magnetic resonance imaging (MRI) are examples of imaging diagnostic systems used to identify important structures of the arterial system and the presence of atherosclerotic plaques. Additionally, the building of three-dimensional (3D) models of the diagnosed arterial system from medical images allows a better envisage of the shape of the artery under analysis and the employment of hemodynamic simulations to evaluate the stress on the vessel wall $[1,2,6,7,8]$.

MRI of carotid arteries has been widely used in several studies to identify atherosclerotic plaques and the associated components in order to analyze the progression of the disease [9]. Since the composition of an atherosclerotic plaque plays an important role in the assessment of the risk of its rupture, as well as for neurological events, the correct characterization of the components allows the determination of the instability of the atherosclerotic lesion. However, the segmentation of the lumen and outer boundaries of the arterial system plays an important role in evaluating the atherosclerotic plaque components, since the atherosclerosis is located between those boundaries.

A fully automatic method for the segmentation of the lumen in MR images of carotid arteries is described in this article. The analysis of the regions present in the input MR image is carried out in order to identify the one that represents the potential lumen of the carotid artery under study. Since the lumen is a low intensity and circular-shaped region in axial black-blood MR images of the carotid artery, the use of a circularity index allows the identification of the region with the maximum roundness that may represents the potential lumen. Then, an active contour method is applied to refine the boundary of the identified region. The results obtained by the proposed method were compared against the corresponding manual delineations in order to evaluate the accuracy of the automatic segmentation. 


\section{Materials and methods}

In this study, the MR images of the carotid artery used in the research of van Engelen et al [10] and provided by the authors under request were selected to perform the experiments. The provided images are regions of interest (ROI) surrounding the carotid arteries. From the original image dataset, 181 postcontrast 3D-T1W images and 181 Proton Density Weighted (PDW) images acquired from thirteen patients were used. In addition to the MR images, masks of the lumen and arterial wall drawn on each slice of the images used in the experiments were also provided. More details about the MRI exams are available in van Engelen et al [10].

The diagram of the proposed method is depicted in Figure 1.

Our method has three main stages: pre-processing, segmentation and lumen identification. The pre-processing stage is adopted to minimize the noisy artifacts and enhance the contrast of the input MR images. Then, the images are submitted to the segmentation stage in order to identify the regions with low grayscale intensities, which include the image background and the lumen regions. The lumen identification stage consists of methods used to identify the region corresponding to the lumen of the carotid artery. The boundary of the identified lumen region is then refined by an active contour algorithm.

In the pre-processing step, the median filter is applied to minimize the noises in the input images, whereas the Adaptive Gamma Correction with Weighted Distribution (AGCWD) proposed by Huang et al [11] is employed to enhance the contrast of dark regions of the images.

In the segmentation step, the combination of the K-means clustering algorithm with the subtractive clustering $[12,13,14]$ is adopted to separate the regions of the enhanced MR images. Segmentation based on the subtractive clustering algorithm was adopted to improve the stability concerning the establishment of the values of the cluster centroids used in the K-means clustering algorithm.

In the subtractive clustering algorithm, the correct number of cluster centroids regarding the characteristics of the image under analysis is calculated from the potential of each pixel in the neighborhood as:

$$
P_{i}=\sum_{j=1}^{n} e^{\frac{-4\left\|x_{i}-x_{j}\right\|^{2}}{r_{a}^{2}}},
$$

where $\left\|x_{i}-x_{j}\right\|$ represents the distance between pixels $x_{i}$ and $x_{j}, r_{a}$ is the radius representing the neighborhood and $n$ is the number of pixels in the input image. Equation 1 gives the initial potential of each pixel; then, the pixel having the highest potential is selected as the first cluster centroid. The next centroids are found according to:

$$
P_{i}=P_{i}-P_{j} \times e^{\frac{-4\left\|x_{i}-x_{j}\right\|^{2}}{r_{b}^{2}}}
$$




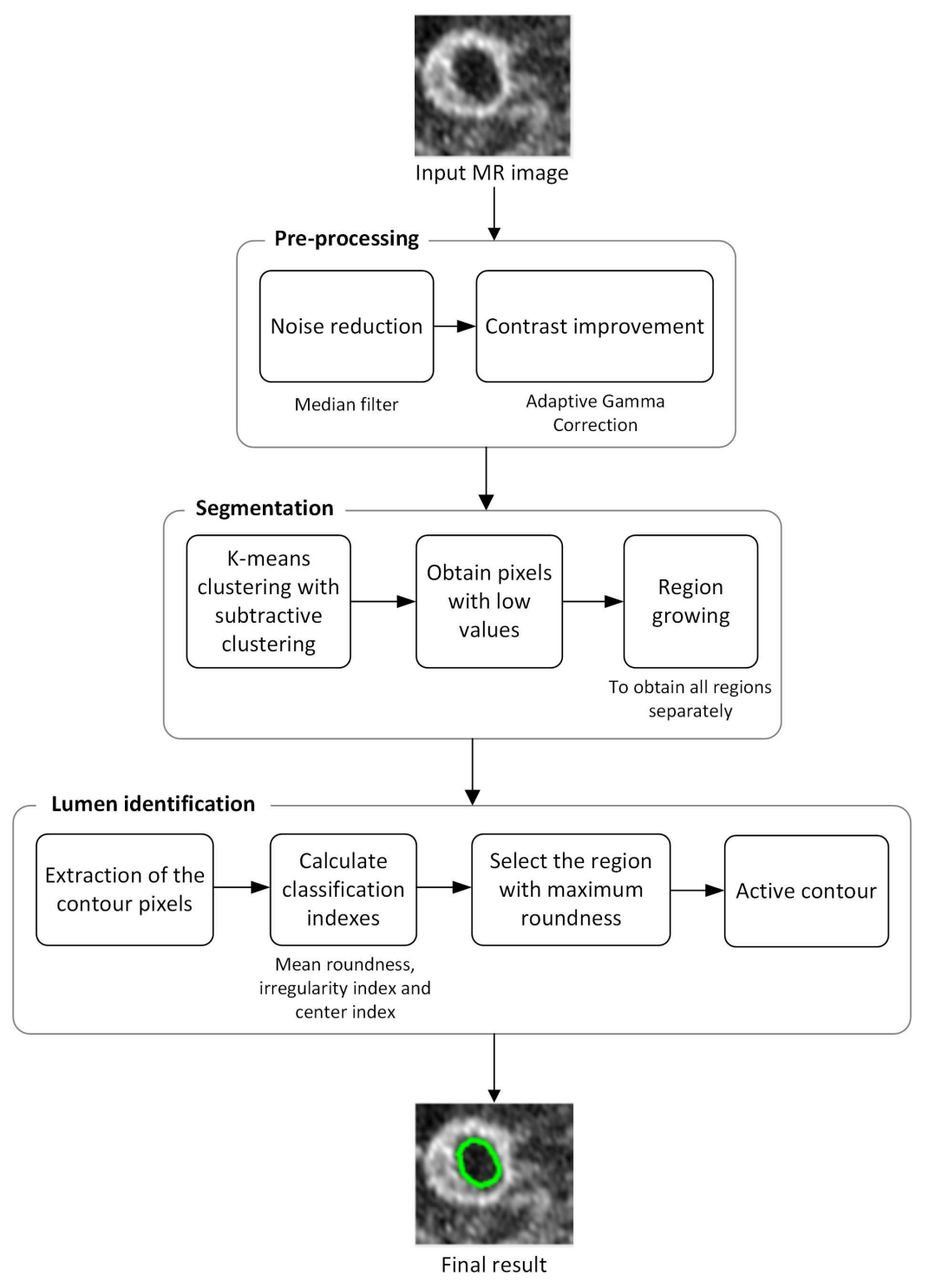

Fig. 1 Diagram of the proposed method.

where $P_{j}$ represents the highest potential, $x_{j}$ is the pixel with highest potential and $r_{b}$ is the radius representing the neighborhood. Equation 2 reduces the potential of the neighbor pixels; then, the next pixel with highest potential is selected as the next cluster centroid and the process is repeated until the number of cluster centroids has been reached. In the proposed method, four cluster centroids are defined and the 
intensity value of each pixel is used in the subtractive clustering and in the K-means algorithm.

Considering the fact that the lumen and background regions are commonly represented by low grayscale intensities in black-blood MR images, the next step consists in identifying the regions belonging to the cluster with low intensity values. A binary image with such regions represented in white and the background represented in balck is then returned and inputted to the region growing algorithm in order to obtain all regions separately, being the white pixels used as seeds of the region growing algorithm. Additionally, regions with less than $1.5 \%$ of the total number of the image pixels are avoided in the next processing steps since such regions are usually associated to noisy artifacts.

The process of identifying the lumen consists in maximizing a function composed by the following indexes:

- Circularity index $(M R)$;

- Irregularity index (Ir); and

- Center index $(d)$.

Circularity indexes have been commonly used to quantify the roundness of regions in images. In this work, the circularity index proposed by Ritter and Cooper [15] is used to find the region with the maximum roundness:

$$
M R=\frac{1}{N} \sum_{i=1}^{N} \frac{\overline{r_{b}}}{\left|r_{i}-\overline{r_{b}}\right|+\overline{r_{b}}}
$$

where $r_{i}$ is the radius of the pixel $i$ with respect to the center of the region under analysis, $r_{b}$ is the average radius and $N$ is the number of pixels [15] of the region contour. The larger is the mean roundness $(M R)$, the more circular is the region.

In addition to the mean roundness, the irregularity index (Ir) is used to avoid regions with irregular contours:

$$
I r=P *\left(\frac{1}{S D}-\frac{1}{G D}\right),
$$

where $P$ is the number of pixels of the contour of the region under analysis, $S D$ is the shortest diameter and $G D$ is the greatest diameter of the same contour [16]. If the difference between $S D$ and $G D$ is equal to 0 (zero) or close to it, the $I r$ decreases. Thus, the boundary is more regular in this case.

In addition to the irregularity index $I r$, a center index $(d)$ is used to avoid regions distant from the center of the input image. Since the lumen is located close to the center of the ROI that surrounds the carotid artery, the distance between the center coordinates of the image and the center coordinates of each segmented region is calculated and used as a term to maximize the proposed circularity index. The combination of the mean roundness defined in Equation 3 and the inverse of the irregularity and center indexes is proposed to maximize the following circularity index: 


$$
E=M R+\frac{1}{I r}+\frac{1}{d}
$$

where $M R$ is the mean roundness, $I r$ is the irregularity index defined in Equation 4 and $d$ is the distance between the center of the image and the center of each segmented region. The additional indexes are used to penalize the mean roundness of regions far from the image's center or having irregular borders. Therefore, regions with higher mean roundness and lower irregularity and center indexes have high probability of being associated to the lumen of the carotid artery.

The Chan-Vese active contour algorithm [17] is then used to refine the contour of the identified lumen region. The binary image with the lumen region represented in white is used in the Chan-Vese active contour algorithm to fit the true boundary of the lumen in the input MR image. Since the gradient of the image is not used, the Chan-Vese active contour was adopted in this study to handle the common weak boundaries of the structures present in medical images.

\section{Results}

The proposed approach was performed on each slice of the postcontrast 3D-T1W and PDW images with the ground truth provided. The Dice coefficient, polyline distance, Hausdorff distance and center distance were used to validate the developed method. The center distance is the difference between the center of the region corresponding to the manual delineation and the center of the region corresponding to the automatically identified lumen. The following parameters were defined for the automatic segmentation of the lumen: the dimension of the mask of the median filter was set to $5 \times 5$; the radius $r_{a}$ and $r_{b}$ used in the subtractive clustering (see Equations 4 and 5 in Bataineh et al [12]) were set to 1.2 and 1.8, respectively; the number of cluster of the K-means algorithm was set to 4 (four); and the number of iterations for further refinament of the lumen contour performed by the Chan-Vese active contour algorithm was set to 200. Examples of segmentation results obtained by the proposed method along with the corresponding manual delineations are shown in Figures 2 and 3.

From Figures 2 and 3, one can notice that in all images the location of the lumen was corrected identified by the automated method.

The error bar plots of the average values of the Dice coefficient, polyline distance, Hausdorff distance and centroid distance of the automatically segmented lumen in comparison to the manual delineations for each set of tested images are shown in Figures 4 and 5, respectively.

The segmentation results obtained from the postcontrast 3D-T1W images were better than the ones obtained from the PDW images, reaching Dice coefficients ranging from $0.67 \pm 0.18$ to $0.91 \pm 0.04$. The lower quality of the PDW images was responsible to decrease the accuracy of the proposed segmentation method. The difference between the automatically detected and manually delineated lumen contours was assessed by using the polyline and Hausdorff distances. For the postcontrast 3D- 


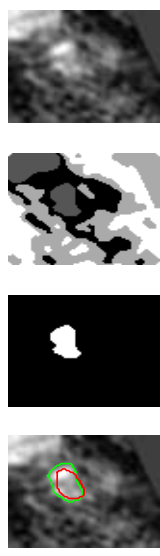

(a)
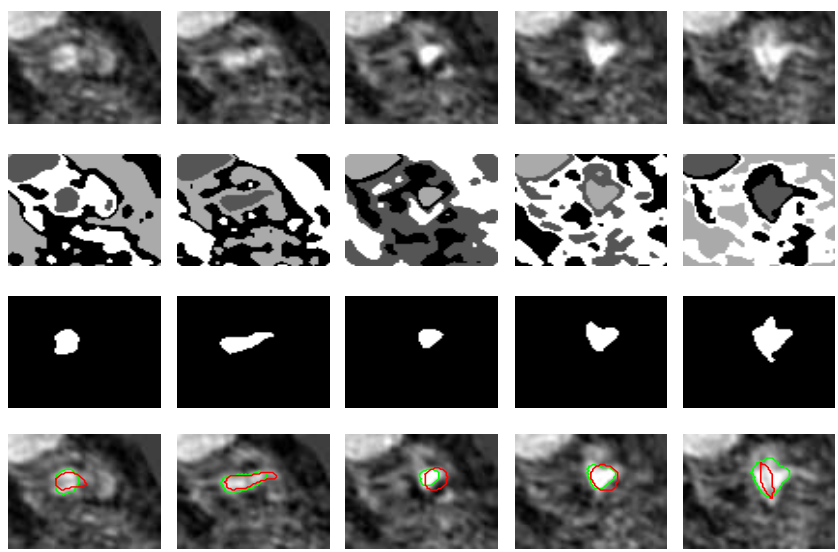

(b)
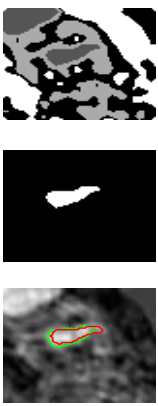

(c)
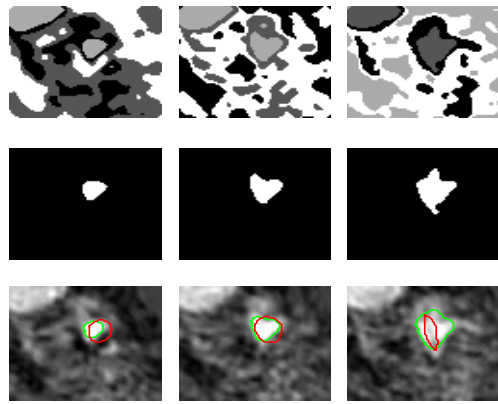

(d)

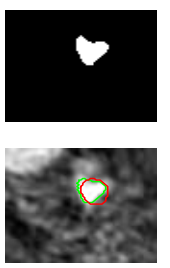

(e)

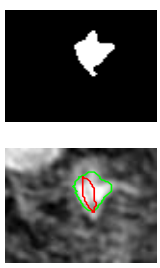

(f)

Fig. 2 Examples of segmentation results obtained from the postcontrast 3D-T1W images: The input MR images are shown in the first row. The results obtained by the K-means clustering algorithm with subtractive clustering are shown in the second row. The third row contains the lumen regions identified by the proposed circularity index. The refined contours of the identified lumen regions along with the corresponding manual delineations are depicted in the forth row. (The contours obtained by the proposed method are shown in green, whereas the contours in red represent the manual delineations.)

$\mathrm{T} 1 \mathrm{~W}$ images, the polyline distance ranged from $1.06 \pm 0.28$ to $4.13 \pm 5.69$ pixels, whereas for the Hausdorff distance, the differences ranged from $2.58 \pm 1.02$ to 9.97 \pm 9.65 pixels. On the other hand, for the PDW images, the distances calculated by the polyline distance ranged from $1.44 \pm 0.59$ to $7.01 \pm 4.98$ pixels, whereas for the Hausdorff distance, the differences ranged from $3.60 \pm 2.07$ to $14.91 \pm 7.62$ pixels.

It is important to notice that the segmentation errors obtained from the images of patient 11 contributed to reduce the Dice coefficient for the PDW images, as shown in the error plot of Figure 5. The presence of noisy artifacts in the PDW images of this patient and the abnormal shape of the lumen regions were responsible for the majority of the incorrect segmentations. Additionally, misalignments between the manual delineations and the real location of the lumen regions might induce incorrect quantitative analysis of the segmentation results.

\section{Conclusions}

The challenges regarding the development of methods for the fully automatic segmentation of the lumen and outer boundaries of the arterial system remains a strong research topic. An automatic segmentation of the lumen in MR images of carotid arteries without user interaction was described in this article. The developed approach 

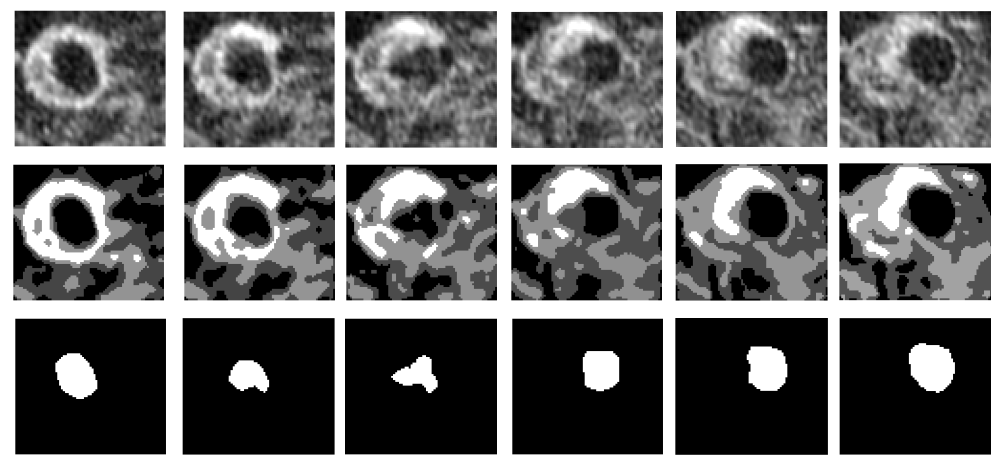

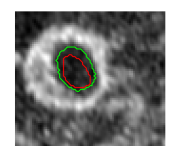

(a)

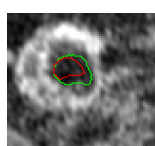

(b)

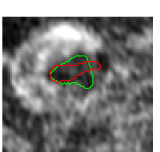

(c)

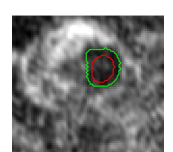

(d)

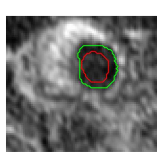

(e)

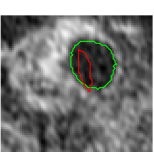

(f)

Fig. 3 Examples of segmentation results obtained from the PDW images: The input MR images are shown in the first row. The results obtained by the K-means clustering algorithm with subtractive clustering are shown in the second row. The third row contains the lumen regions identified by the proposed circularity index. The refined contours of the identified lumen regions along with the corresponding manual delineations are depicted in the forth row. (The contours obtained by the proposed method are shown in green, whereas the contours in red represent the manual delineations.)
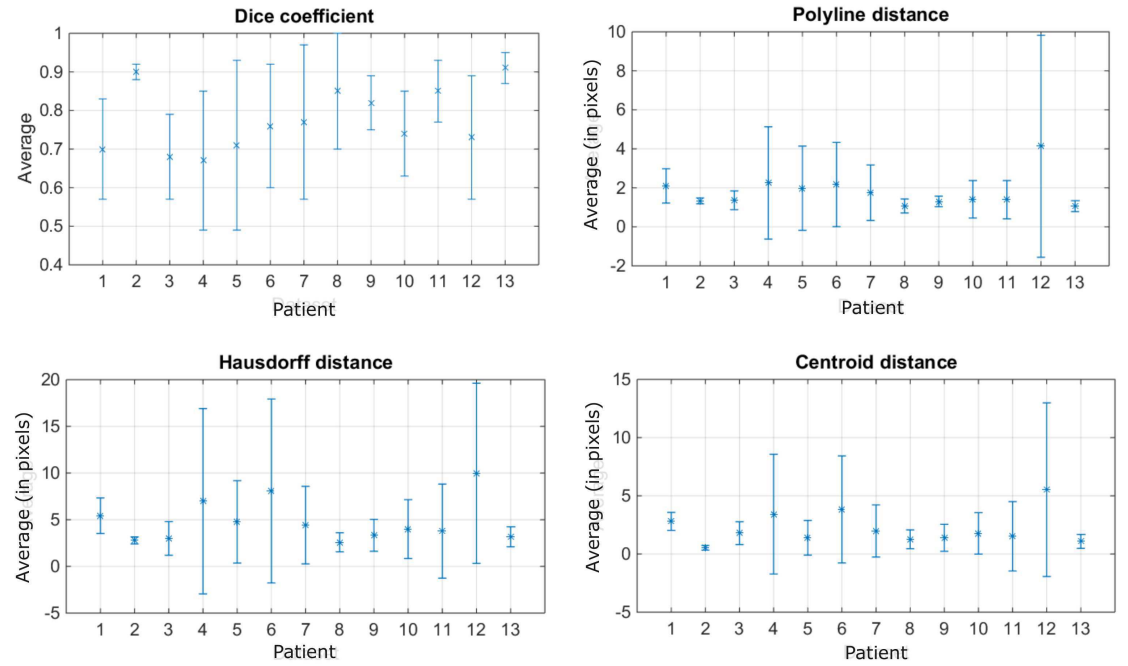

Fig. 4 Error bar plots with the average values (marked with an ' $x$ ') and standard deviation of the Dice coefficient, polyline distance, Hausdorff distance and center distance obtained for the postcontrast 3D-T1W images. 

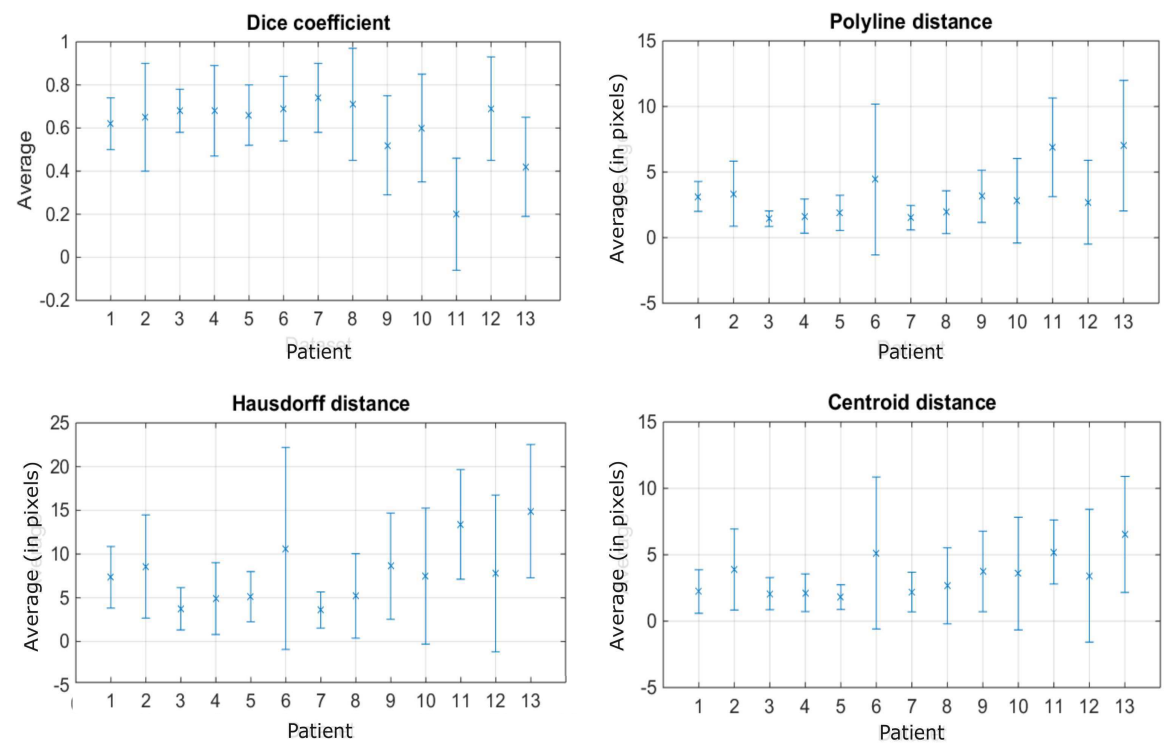

Fig. 5 Error bar plots with the average values (marked with an ' $x$ ') and standard deviation of the Dice coefficient, polyline distance, Hausdorff distance and center distance obtained for the PDW images.

proved to be effective in identifying the correct location of the lumen in MR images. In spite of the segmentation errors induced by the low quality of the input image and malformation of the lumen regions, the results showed high overlap and low contour distances between the automatically segmented lumen and the correspondent manual delineation. The reduction of the number of parameters used in the developed algorithm is expected to be addressed in future studies. Additionally, the segmentation of the outer boundary of the carotid artery is expected to be performed in the next step of our research work.

Acknowledgements This work was partially funded by "Coordenação de Aperfeiçoamento de Pessoal de Nível Superior" (CAPES), funding agency in Brazil, under the PhD Grant with reference 0543/13-6.

The authors thank the funding of Project NORTE-01-0145-FEDER-000022 - SciTech - Science and Technology for Competitive and Sustainable Industries, co-financed by "Programa Operacional Regional do Norte" (NORTE2020), through "Fundo Europeu de Desenvolvimento Regional" (FEDER).

\section{References}

[1] Sousa LC, Castro CF, António CC, Santos A, Santos R, Castro P, Azevedo E, Tavares JMRS (2014) Haemodynamic conditions of patient-specific carotid 
bifurcation based on ultrasound imaging. Computer Methods in Biomechanics and Biomedical Engineering: Imaging \& Visualization pp 157-166

[2] Sousa LC, Castro CF, António CC, Santos AMF, Santos RM, Castro PMAC, Azevedo E, Tavares JMRS (2014) Toward hemodynamic diagnosis of carotid artery stenosis based on ultrasound image data and computational modeling. Medical \& Biological Engineering \& Computing 52(11):971-983

[3] Furie KL, Smimakis SM, Koroshetz WJ, Kistler JP (2004) Stroke due to large artery atherosclerosis. In: Furie KL, Kelly PJ (eds) Handbook of Stroke Prevention in Clinical Practice, Current Clinical Neurology, Humana Press, pp $151-165$

[4] Schadé JP (2006) The Complete Encyclopedia of Medicine \& Health. Foreign Media Books

[5] Wiebers DO, Feigin VL, Brown R (2006) Handbook of Stroke, 2nd edn. Board Review Series, Lippincott Williams \& Wilkins, Philadelphia, PA

[6] Cheng GC, Loree HM, Kamm RD, Fishbein MC, Lee RT (1993) Distribution of circumferential stress in ruptured and stable atherosclerotic lesions. A structural analysis with histopathological correlation. Circulation 87(4):1179-1187

[7] Huang H, Virmani R, Younis H, Burke AP, Kamm RD, Lee RT (2001) The impact of calcification on the biomechanical stability of atherosclerotic plaques. Circulation 103(8):1051-1056

[8] Yulia K, Ivanov DV, Dol AV (2016) Biomechanical bases of forecasting occurrence of carotid atherosclerosis. Annals of Circulation 1(1):001-006

[9] Jodas DS, Pereira AS, Tavares JMR (2016) A review of computational methods applied for identification and quantification of atherosclerotic plaques in images. Expert Systems with Applications 46:1-14

[10] van Engelen A, Niessen WJ, Klein S, Groen HC, Verhagen HJM, Wentzel JJ, van der Lugt A, de Bruijne M (2014) Atherosclerotic plaque component segmentation in combined carotid MRI and CTA data incorporating class label uncertainty. PLoS ONE 9(4):1-14

[11] Huang SC, Cheng FC, Chiu YS (2013) Efficient Contrast Enhancement Using Adaptive Gamma Correction with Weighting Distribution. IEEE Transactions on Image Processing 22(3):1032-1041

[12] Bataineh K, Naji M, Saqer M (2011) A comparison study between various fuzzy clustering algorithms. Editorial Board 5(4):335-343

[13] Chiu SL (1994) Fuzzy model identification based on cluster estimation. Journal of Intelligent \& Fuzzy Systems 2(3):267-278

[14] Dhanachandra N, Manglem K, Chanu YJ (2015) Image Segmentation Using K -means Clustering Algorithm and Subtractive Clustering Algorithm. Procedia Computer Science 54:764-771

[15] Ritter N, Cooper J (2009) New Resolution Independent Measures of Circularity. Journal of Mathematical Imaging and Vision 35(2):117-127

[16] Jain S, Jagtap V, Pise N (2015) Computer Aided Melanoma Skin Cancer Detection Using Image Processing. Procedia Computer Science 48:735-740

[17] Chan TF, Vese LA (2001) Active contours without edges. IEEE Transactions on Image Processing 10(2):266-277 